\title{
Desenvolvimento de Oligonychus ilicis em Coffea canephora sob diferentes temperaturas
}

\author{
Ricardo Antonio Polanczyk ( ${ }^{(*)}$; Flávio Neves Celestino ( $\left.{ }^{2}\right)$; Lígia Souza Ferreira (3); \\ Débora Ferreira Melo (2); Luziani Rezende Bestete (2); Cláudio Roberto Franco ( $\left.{ }^{4}\right)$; Dirceu Pratissoli ( ${ }^{2}$ ) \\ (') Universidade Estadual Paulista "Júlio de Mesquita Filho" (UNESP/FCAV), 14884-900 Jaboticabal (SP). \\ (2) Universidade Federal do Espírito Santo, Centro de Ciências Agrárias, 29800-000 Alegre (ES). \\ (3) Universidade Estadual do Norte Fluminense, 28010-970 Campus dos Goytacazes (RJ). \\ (4) Universidade do Estado de Santa Catarina, 88501-900 Lages (SC). \\ (*) Autor correspondente: rapolanc@fcav.unesp.br
}

Recebido: 10/mai./2009; Aceito: 24/ago./2010

\section{Resumo}

Oligonychus ilicis (McGregor) (Acari) (Tetranychidae) é uma das principais pragas de Coffea canephora Pierre \& Froehner. Neste trabalho, avaliou-se o efeito de diferentes temperaturas no desenvolvimento desse ácaro-vermelho em folhas de C. canephora, em laboratório. Confinaram-se 80 fêmeas em 40 arenas, constituídas de disco foliar de 4 cm, para oviposição nas temperaturas de 21, 24, 27, 30 e $33^{\circ} \mathrm{C}$. Foram selecionados ao acaso pelo menos dois ovos para avaliar o desenvolvimento embrionário. Após a eclosão da larva, foram realizadas avaliações a cada 12 horas para obtenção da duração e sobrevivência larval. Para avaliar a longevidade foi transferido um ácaro macho da criação para as arenas com uma fêmea para acasalamento. O limite térmico de desenvolvimento inferior e a constante térmica foram determinados para a duração do desenvolvimento de ovo a adulto. O tempo de desenvolvimento das fases imaturas diminuiu com o aumento da temperatura. As fases de ovo, larva, protocrisálida, protoninfa, deutocrisálida, deutoninfa e teleiocrisálida variaram, respectivamente, de 10,4 a 4,3; 2,4 a 1,2; 2,0 a 1,0; 2,3 a 1,2; 1,9 a 1,0; 3,0 a 1,5 e 2,3 a 1,0 dias. O aumento da temperatura afetou o período de desenvolvimento, reduzindo a duração de ovo-adulto e a longevidade. O limite térmico inferior foi de $9,0{ }^{\circ} \mathrm{C}$ para o período de ovo-adulto e constante térmica de 256,4 graus-dias. Em condições de laboratório, a faixa de temperatura que favorece o desenvolvimento do ácaro em C. canephora foi de 24 a $30^{\circ} \mathrm{C}$. O limite térmico inferior não é limitante para ocorrência de O. ilicis em áreas cultivadas com C. canephora no estado do Espírito Santo.

Palavras-chave: Acari, Tetranychidae, exigências térmicas, constante térmica, cafeeiro, Conillon.

\section{Development of Oligonychus ilicis on Coffea canephora under different temperatures}

\section{Abstract}

Oligonychus ilicis (McGregor) (Acari) (Tetranychidae) is one of the most important pests of Coffea canephora Pierre \& Froehner. In this study, the effect of different temperatures in the development of red mite in C. canephora leaves was evaluated. Eighty adult females were placed in 40 cages to oviposition containing $4 \mathrm{~cm}$ leaf discs at 21, 24, 27,30 and $33{ }^{\circ} \mathrm{C}$. To evaluated embrionary development two randomly selected eggs were allowed to complete development. After larvae hatch, evaluations were performed each 12 hours to determine the survival and larval period. To evaluate longevity one male was transferred to a cage with one female to mate. The thermal threshold of development and thermic constant were determined to egg adult period of development. The development period of immature stages of 0 . ilicis decreased as soon as the temperature increased. The stages of egg, larvae, protochrysalidae, protonymph, deutochrysalidae, deutonymph and teleiochrysalidae ranged from 10.4 to $4.3,2.4$ to $1.2,2.0$ to $1.0,2.3$ to $1.2,1.9$ to $1.0,3.0$ to 1.5 and 2.3 to 1.0 days, respectivelly. The temperature increase affected the period of egg adult development and adult longevity The thermal threshold of development was $9.0^{\circ} \mathrm{C}$ to egg-adult period and thermal constant was 256.4 GD. At laboratory conditions, temperatures between 24 and $30^{\circ} \mathrm{C}$ favours the mite development in C. canephora. The thermal threshold of development does not constrain the 0 . ilicis occurrence with $C$. canephora under field conditions in the State of Espirito Santo, Brazil.

Key words: Acari, Tetranychidae, thermal requirements, thermal constant, coffee plant, Conillon. 


\section{INTRODUÇÃO}

O Brasil é o maior produtor e exportador mundial de café e o Estado do Espírito Santo (ES) é o segundo maior produtor do Brasil, sendo o cafeeiro Conillon Coffea canephora Pierre \& Froehner assume maior relevância. Na safra 2008, no Espírito Santo foram produzidas 7,363 milhóes de sacas de café Conillon, o que representa $72 \%$ da produção estadual de café (ConAB, 2010).

O cafeeiro é atacado por diversas pragas, destacando-se o ácaro-vermelho do cafeeiro Oligonychus ilicis (McGregor) (Acari: Tetranychidae), que já foi referido como a segunda praga em importância para o cafeeiro Conillon no Estado do Espírito Santo, sendo essa planta considerada mais sensível ao ácaro do que o cafeeiro Arábica (C. arabica L.) (Reis et al., 2004). Esse ácaro se alimenta principalmente na face superior das folhas, perfurando as células e sugando o conteúdo celular, cobrindo-as com uma delicada teia, onde aderem detritos, poeira e suas exúvias, dando às folhas um aspecto de sujidade. Em consequência, as folhas perdem o brilho natural, tornam-se bronzeadas e caem, reduzindo a capacidade fotossintética da planta (ReIs e ZaCarias, 2007). $\mathrm{O}$ ataque ocorre geralmente em reboleiras, porém, em longo período de estiagem e falta de manejo adequado, poderá atingir toda a lavoura, causando desfolha e provocando atraso no desenvolvimento de plantas jovens (Reis e Souza, 1986; Reis, 2005).

A temperatura é o mais importante fator ambiental que afeta a população de ácaros da família Tetranychidae (Silva, 2002). Altas temperaturas promovem decréscimo na longevidade e aumento na taxa de oviposição e fecundidade em diversas espécies (SiLVA, 2002; VAsCONCELOS et al., 2004). Os limites da temperatura a partir dos quais os ácaros deixam de sobreviver variam muito com as espécies e com sua fase de desenvolvimento (Boudreaux, 1963; Bonato, 1999).

Tanto para as plantas como para os artrópodes, as velocidades de desenvolvimento estáo correlacionadas com o número de graus de temperatura que se acumulam durante determinado período, ou seja, com o somatório de unidades térmicas denominadas graus-dia. Este somatório de unidades, que considera apenas valores da temperatura acima do zero de desenvolvimento, é um fator de fundamental importância para determinar os efeitos da temperatura sobre os artrópodes, e principalmente sobre o sincronismo que se estabelece entre as diferentes fases do desenvolvimento desses artrópodes-praga e seus hospedeiros. A relação empírica entre a temperatura e a taxa de desenvolvimento embrionário e pós-embrionário dos ácaros tetraniquídeos aumenta até um ponto máximo, independentemente da temperatura e diminui a partir deste ponto (tendência curvilínea). Assim, podem ser estimados os valores de temperatura-base e constante térmica desses ácaros, através da linearização da curva obtida em laboratório (Silva, 2002). Desta forma, o objetivo deste trabalho foi avaliar o efeito de diferentes temperaturas no desenvolvimento $O$. ilicis em $C$. canephora com a finalidade de prever os picos populacionais desta praga.

\section{MATERIAL E MÉTODOS}

\section{Criação de 0 . ilicis}

A criaçáo de $O$. ilicis foi iniciada com fêmeas adultas coletadas em plantas de $C$. canephora cultivadas em Alegre (ES). Os ácaros coletados foram mantidos em arenas confeccionadas com folhas de C. canephora. Essas folhas foram acondicionadas sobre discos de manta acrílica de 18 $\mathrm{cm}$ de diâmetro no interior de bandejas plásticas de 20 $\mathrm{cm}$ de diâmetro, sendo a folha circundada com algodão hidrófilo, umedecido com água destilada para manter a folha túrgida e evitar a fuga dos ácaros. Os ácaros foram semanalmente transferidos para novas arenas, sendo observada razão sexual de 0,5 . A criação foi mantida em sala climatizada a $25 \pm 2{ }^{\circ} \mathrm{C}$, umidade relativa de $60 \pm 10 \%$ e fotofase de 14 horas.

\section{Desenvolvimento de $\boldsymbol{0}$. ilicis}

Em arenas com discos foliares de $4 \mathrm{~cm}$ diâmetro da variedade Incaper 8142 (Conillon Vitória) clone 3, sobrepostas a um disco de manta acrílica de $6 \mathrm{~cm}$ de diâmetro e circundadas por uma camada de algodão hidrófilo umedecida com água destilada, foi avaliada a oviposição de $O$. ilicis, durante 12 horas, em câmaras climatizadas às temperaturas de $21,24,27,30$ e $33{ }^{\circ} \mathrm{C}$ (totalizando 40 arenas para cada temperatura), umidade relativa de $60 \pm 10 \%$ e fotofase de 12 horas. Após a oviposição, as fêmeas (média 14 ovos/fêmea) foram retiradas e os ovos mantidos em suas respectivas temperaturas, e em cada arena foram deixados pelo menos dois ovos. Após a eclosão da larva, as avaliações foram realizadas em intervalos de 12 horas para determinar o período de desenvolvimento embrionário, duração e sobrevivência das fases de larva, protocrisálida, protoninfa, deutocrisálida, deutoninfa e teleiocrisálida. Para avaliar a longevidade, após a emergência dos adultos, foi transferido um ácaro macho proveniente da criação para as arenas que tinham uma fêmea, para acasalamento. Em caso de morte, o macho era substituído por outro da criação. As avaliações foram realizadas em intervalos de 12 horas. A postura realizada nas primeiras 24 horas do período de oviposição foi utilizada para determinar a viabilidade dos ovos. As arenas foram umedecidas com água destilada, duas vezes ao dia, para manter as folhas túrgidas e evitar a fuga de ácaros. Os discos de folhas foram trocados a cada sete dias ou quando necessário. 
Os dados foram analisados sem a separação pelo sexo do ácaro, adotando-se o delineamento experimental inteiramente casualizado, com cinco tratamentos representados pelas temperaturas. Para cada parâmetro avaliado, os resultados foram submetidos à análise de regressão polinomial, significativo a $5 \%(\mathrm{p}<0,05)$.

\section{Exigências térmicas}

O limite térmico de desenvolvimento inferior $(\mathrm{Tb})$ e a constante térmica $(\mathrm{K})$ foram determinados para a duração do desenvolvimento de ovo a adulto, através do método da hipérbole (HADDAD et al., 1999).

\section{RESULTADOS E DISCUSSÃO}

\section{Desenvolvimento de $\mathbf{0}$. ilicis}

O tempo de desenvolvimento das fases imaturas de O. ilicis, em folhas de cafeeiro Conillon C. canephora variedade Incaper 8142 (Conillon Vitória) clone 3, diminuiu progressivamente com o aumento da temperatura. A duração dos estágios de ovo, larva, protocrisálida, protoninfa, deutocrisálida, deutoninfa e teleiocrisálida de $O$. ilicis, de 21 a $33{ }^{\circ} \mathrm{C}$, variou de 10,4 a 4,$3 ; 2,4$ a 1,$2 ; 2,0$ a $1,0,2,3$ a
$1,2,1,9$ a $1,0,3,0$ a 1,5 e 2,3 a 1,0 dias respectivamente (Tabela 1). A redução na duração dos estágios imaturos de outras espécies de ácaros, em função do aumento da temperatura, também foi verificada por VASCONCELOS et al. (2004) e Ferreira et al. (2006) entre as faixas térmicas de 18 e $32{ }^{\circ} \mathrm{C}$. O modelo matemático que melhor expressou esta relação foi o quadrático, com coeficiente de determinação superior a 44,7\% (Tabela 1). O período de desenvolvimento de ovo a adulto variou de 24,6 a 11,5 dias entre as temperaturas de 21 e $33^{\circ} \mathrm{C}$ (Tabela 1). SiLVA (2002) e VAsCONCELOS et al. (2004) constataram resultados semelhantes para Tetranychus ludeni Zacher e T. abacae Baker \& Printchard (Tetranychidae) respectivamente. As duraçóes de ovo a adulto observadas nas temperaturas de 27 e $30^{\circ} \mathrm{C}(12,7$ e 11,7 dias $)$ foram semelhantes às observadas por ReIs et al. (1997) para O. ilicis em folhas de $C$. arábica: de 11,6 e 11,8 dias, respectivamente, para fêmeas e machos na temperatura de $25 \pm 2{ }^{\circ} \mathrm{C}$.

O acréscimo de temperatura também reduziu a longevidade dos adultos, variando entre 8,5 e 2,3 dias respectivamente nas temperaturas de 21 e $33^{\circ} \mathrm{C}$ e para esse parâmetro o modelo matemático adequado foi o linear (Tabela 1 ). A relação entre a duração do ciclo total de desenvolvimento e a temperatura pode ser descrita pelo modelo matemático quadrático $\left(\mathrm{R}^{2}=0,70\right)$; a duração do ciclo total de $O$. ilicis variou de 33,1 a 13,8 dias para as temperaturas entre $21 \mathrm{e}$ $33{ }^{\circ} \mathrm{C}$ respectivamente, verificando-se também a redução

Tabela 1. Duração* (dias \pm erro padrão da média) dos estágios embrionário e pós-embrionários de Oligonychus ilicis em folhas de Coffea canephora variedade Incaper 8142 (Conillon Vitória) clone 3, em diferentes temperaturas

\begin{tabular}{|c|c|c|c|c|c|c|c|}
\hline \multirow{2}{*}{$\begin{array}{l}\text { Estágio de } \\
\text { desenvolvimento }\end{array}$} & \multicolumn{5}{|c|}{ Temperatura $\left({ }^{\circ} \mathrm{C}\right) /$ Duração em dias dos diversos estágios do desenvolvimento } & \multicolumn{2}{|c|}{$\begin{array}{l}\text { Equação da } \\
\text { regressão }\end{array}$} \\
\hline & 21 & 24 & 27 & 30 & 33 & Dias & $\mathbf{R}^{2}(\%)$ \\
\hline Ovo & $\begin{array}{c}10,4 \pm 0,14 \\
(8,0-12,5)[35]\end{array}$ & $\begin{array}{c}6,6 \pm 0,07 \\
(6,0-7,5)[32]\end{array}$ & $\begin{array}{c}4,8 \pm 0,09 \\
(2,5-5,5)[37]\end{array}$ & $\begin{array}{c}4,3 \pm 0,09 \\
(3,5-6,0)[36]\end{array}$ & $\begin{array}{c}4,3 \pm 0,09 \\
(3,5-5,5)[28]\end{array}$ & $\begin{array}{c}68,985- \\
4,270 T+ \\
0,070 T^{2}\end{array}$ & 93,7 \\
\hline Larva & $\begin{array}{c}2,4 \pm 0,11 \\
(1,0-4,0)[35]\end{array}$ & $\begin{array}{c}1,7 \pm 0,06 \\
(1,0-2,5)[32]\end{array}$ & $\begin{array}{c}1,4 \pm 0,05 \\
(1,0-2,0)[37]\end{array}$ & $\begin{array}{c}1,4 \pm 0,07 \\
(1,0-3,0)[36]\end{array}$ & $\begin{array}{c}1,2 \pm 0,05 \\
(1,0-2,0)[28]\end{array}$ & $\begin{array}{c}11,047- \\
0,622 T+ \\
0,010 T^{2}\end{array}$ & 44,7 \\
\hline Protocrisálida & $\begin{array}{c}2,0 \pm 0,07 \\
(1,5-2,5)[27]\end{array}$ & $\begin{array}{c}1,5 \pm 0,058 \\
(1,0-2,0)[25]\end{array}$ & $\begin{array}{c}1,1 \pm 0,04 \\
(1,0-1,5)[35]\end{array}$ & $\begin{array}{c}1,1 \pm 0,05 \\
(1,0-2,0)[33]\end{array}$ & $\begin{array}{c}1,0 \pm 0,00 \\
(1,0-1,0)[5]\end{array}$ & $\begin{array}{c}10,753- \\
0,640 T+ \\
0,011 T^{2}\end{array}$ & 60,8 \\
\hline Protoninfa & $\begin{array}{c}2,3 \pm 0,10 \\
(1,0-3,0)[21]\end{array}$ & $\begin{array}{c}1,5 \pm 0,07 \\
(1,0-2,5)[23]\end{array}$ & $\begin{array}{c}1,3 \pm 0,05 \\
(1,0-2,0)[33]\end{array}$ & $\begin{array}{c}1,2 \pm 0,05 \\
(1,0-2,0)[30]\end{array}$ & $\begin{array}{c}1,3 \pm 0,14 \\
(1,0-1,5)[4]\end{array}$ & $\begin{array}{c}14,372- \\
0,904 T+ \\
0,015 T^{2}\end{array}$ & 56,1 \\
\hline Deutocrisálida & $\begin{array}{c}1,9 \pm 0,07 \\
(1,5-2,5)[19]\end{array}$ & $\begin{array}{c}1,6 \pm 0,08 \\
(1,0-2,5)[21]\end{array}$ & $\begin{array}{c}1,1 \pm 0,04 \\
(1,0-1,5)[28]\end{array}$ & $\begin{array}{c}1,1 \pm 0,04 \\
(1,0-1,5)[27]\end{array}$ & $\begin{array}{c}1,0 \pm 0,00 \\
(1,0-1,0)[4]\end{array}$ & $\begin{array}{c}8,809- \\
0,492 T+ \\
0,008 T^{2}\end{array}$ & 55,1 \\
\hline Deutoninfa & $\begin{array}{c}3,0 \pm 0,14 \\
(2,0-3,5)[12]\end{array}$ & $\begin{array}{c}1,8 \pm 0,10 \\
(1,0-2,5)[19]\end{array}$ & $\begin{array}{c}1,5 \pm 0,08 \\
(1,0-2,5)[25]\end{array}$ & $\begin{array}{c}1,5 \pm 0,08 \\
(1,0-2,0)[23]\end{array}$ & $\begin{array}{c}1,5 \pm 0,20 \\
(1,0-2,0)[4]\end{array}$ & $\begin{array}{c}21,281- \\
1,375 T+ \\
0,024 T^{2}\end{array}$ & 58,1 \\
\hline Teleiocrisálida & $\begin{array}{c}2,3 \pm 0,11 \\
(2,0-3,0)[12]\end{array}$ & $\begin{array}{c}1,6 \pm 0,06 \\
(1,0-2,0)[19]\end{array}$ & $\begin{array}{c}1,4 \pm 0,06 \\
(1,0-2,0)[24]\end{array}$ & $\begin{array}{c}1,0 \pm 0,03 \\
(1,0-1,5)[23]\end{array}$ & $\begin{array}{c}1,0 \pm 0,00 \\
(1,0-1,0)[2]\end{array}$ & $\begin{array}{c}9,876- \\
0,536 \mathrm{~T}+ \\
0,008 \mathrm{~T}^{2}\end{array}$ & 65,2 \\
\hline Ovo a adulto & $\begin{array}{c}24,6 \pm 0,31 \\
(23,0-27,0)[12]\end{array}$ & $\begin{array}{c}16,5 \pm 0,18 \\
(15,0-17,5)[16]\end{array}$ & $\begin{array}{c}12,7 \pm 0,11 \\
(11,5-13,5)[22]\end{array}$ & $\begin{array}{c}11,7 \pm 0,18 \\
(10,0-13,5)[23]\end{array}$ & $\begin{array}{c}11,5 \pm 0,50 \\
(11,0-12,0)[2]\end{array}$ & $\begin{array}{c}161,738- \\
0,122 T+ \\
0,170 T^{2}\end{array}$ & 96,5 \\
\hline Longevidade & $\begin{array}{c}8,5 \pm 1,01 \\
(4,0-14,0)[12]\end{array}$ & $\begin{array}{c}8,2 \pm 1,03 \\
(3,5-19,0)[16]\end{array}$ & $\begin{array}{c}8,1 \pm 0,95 \\
(1,5-15,5)[22]\end{array}$ & $\begin{array}{c}5,5 \pm 0,56 \\
(1,0-13,5)[23]\end{array}$ & $\begin{array}{c}2,3 \pm 0,75 \\
(1,5-3,0)[2]\end{array}$ & $\begin{array}{c}17,087- \\
0,373 T\end{array}$ & 10,2 \\
\hline
\end{tabular}

* Entre parênteses: valor mínimo e máximo observado; Entre colchetes: número de observaçóes. 
do tempo de desenvolvimento em funçáo do aumento da temperatura (Figura 1).

A sobrevivência das formas imaturas foi de $34,3 \%$ a $21{ }^{\circ} \mathrm{C} ; 50 \%$ a $24{ }^{\circ} \mathrm{C} ; 59,5 \%$ a $27^{\circ} \mathrm{C} ; 63,9 \%$ a $30{ }^{\circ} \mathrm{C}$ e $7,1 \%$ a $33^{\circ} \mathrm{C}$. Essa sobrevivência foi baixa quando comparada com os resultados observados por SiLVA (2002) e VAsConcelos et al. (2004) para outras espécies de Tetranychidae. A viabilidade dos ovos depositados nas 24 horas iniciais do período de oviposição foi de $83 \%$ a 21 ${ }^{\circ} \mathrm{C} ; 88 \%$ a $24^{\circ} \mathrm{C} ; 79 \%$ a $27{ }^{\circ} \mathrm{C} ; 100 \%$ a $30{ }^{\circ} \mathrm{C}$ e $0 \%$ a $33{ }^{\circ} \mathrm{C}$. A partir desses resultados, observa-se que a temperatura de $33{ }^{\circ} \mathrm{C}$ foi desfavorável ao desenvolvimento do $O$. ilicis, uma vez que somente $7,1 \%$ dos espécimes completaram o ciclo de ovo a adulto e estes não deixaram descendentes. Esses resultados são condizentes com a faixa ótima de temperatura $\left(24\right.$ a $\left.29^{\circ} \mathrm{C}\right)$ de desenvolvimento determinada por BoudREAUX (1963) para diversas espécies de tetraniquídeos. Segundo Aponte e McMur-

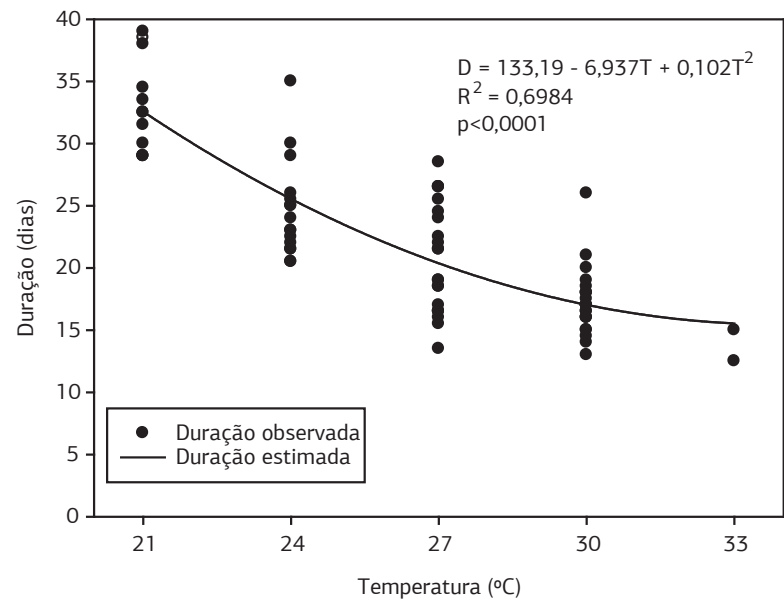

Figura 1. Duraçáo do ciclo total de desenvolvimento, de ovo até a morte do adulto, de Oligonychus ilicis em folhas de Coffea canephora, variedade Incaper 8142 (Conillon Vitória) clone 3.

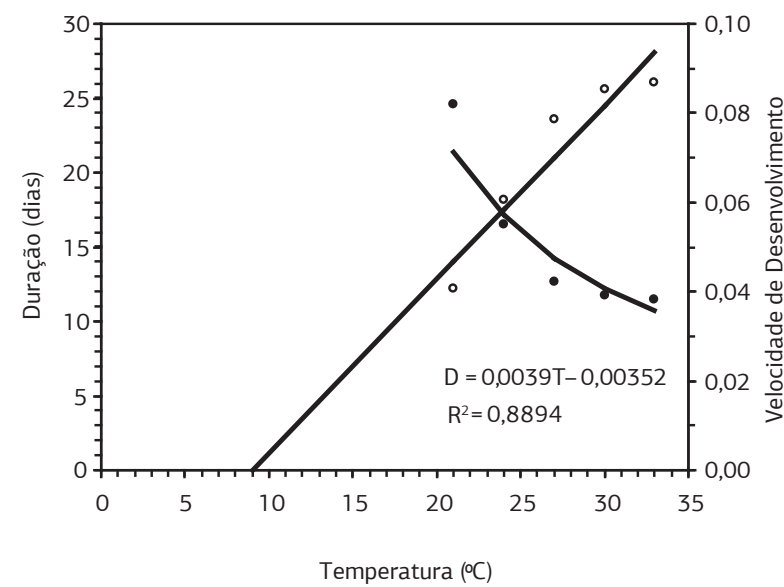

Figura 2. Relação entre temperatura, duração (dias) $(\bullet)$ e velocidade de desenvolvimento (o), de ovo a adulto, de Oligonychus ilicis em folhas de Coffea canephora, variedade Incaper 8142 (Conillon Vitória) clone 3. A temperatura base ( $\mathrm{Tb}$ ), ou limite térmico inferior, foi estimada em 9,0 ${ }^{\circ} \mathrm{C}$ e constante térmica (K) de 256,4 graus-dia.
TRY (1997), o desenvolvimento de O. perseae em abacate também foi inversamente proporcional à temperatura e também verificaram maior mortalidade $(37 \%)$ durante o desenvolvimento na temperatura de $30^{\circ} \mathrm{C}$.

\section{Exigências térmicas}

O modelo matemático mais adequado para descrever a relação entre a temperatura e a velocidade de desenvolvimento de $O$. ilicis foi o linear $\left(\mathrm{R}^{2}=0,89\right)$ e o limite térmico inferior $(\mathrm{Tb})$ foi de $9,0{ }^{\circ} \mathrm{C}$ para o período de ovo a adulto, com a constante térmica (K) de 256,4 graus-dias (Figura 2). Esses resultados foram próximos aos observados por Aponte e McMurtry (1997) para O. perseae, cujos valores de Tb para fêmeas e machos foram de 7,8 e $9,0^{\circ} \mathrm{C}$ respectivamente, enquanto a constante térmica foi de 200 e 166 graus-dias respectivamente. Como a faixa de temperatura ideal para o cultivo de C. canephora está entre 22 e $26^{\circ} \mathrm{C}$ (Matiello, 1991), a baixa temperatura, inferior a $22^{\circ} \mathrm{C}$, não é fator limitante para o crescimento populacional de $O$. ilicis, podendo esta faixa de temperatura ser considerada ideal para o desenvolvimento desta espécie. A obtenção de K é de grande importância pelo fato de a temperatura ser considerada um dos fatores abióticos que mais influenciam a velocidade de desenvolvimento, comportamento, alimentação, fecundidade e a dispersão de insetos e ácaros, bem como fornece informaçôes relevantes sobre o planejamento da criação desses organismos, visando desenvolver atividades de pesquisa (Ferreira et al., 2006).

No Espírito Santo, os principais municípios produtores de café Conillon são Jaguaré, Sooretama, Vila Valério, São Mateus, Rio Bananal e Pinheiros (Fassio e Silva, 2007). Nesses municípios, de acordo com a série histórica de temperatura no mês mais quente, fevereiro, a média diária é de $27^{\circ} \mathrm{C}$ e no mês mais frio, julho, de $22^{\circ} \mathrm{C}$ (CECAM, 2010). Com base nas séries históricas, as temperaturas nos meses de setembro a maio são ótimas para o desenvolvimento de O. ilicis. Entretanto, os meses de junho a agosto também estão sujeitos à ocorrência dessa praga, mesmo que seu desenvolvimento seja mais lento. Este fato pode contribuir para que nos meses seguinte ocorram rápidos surtos populacionais de $O$. ilicis. $\mathrm{O}$ monitoramento intensivo deste ácaro, de setembro a maio, é fundamental nestes municípios, pois as condições climáticas potencializam os danos que podem ser causados por sua infestação.

Precipitaçôes pluviais intensas podem reduzir as populaçôes de O. ilicis, pelo fato de o ácaro habitar a superfície superior das folhas, tecer teias com fios dispersos e os ambúlacros têm menor capacidade de fixação, ficando mais sujeitos ao efeito mecânico da chuva (Franco et al., 2008). Por esse motivo,, a maior ocorrência de $O$. ilicis ocorre na época de menor precipitaçáo pluvial. 
Outro fato importante relaciona-se às mudanças climáticas, visto que existe uma perspectiva de aumento de até $3{ }^{\circ} \mathrm{C}$ na média nos próximos 30 anos (FAzuoLI et al., 2007). Diante dessa perspectiva, outro possível quadro seria que áreas hoje adequadas, poderão no futuro ser inadequadas ( $>30$ graus) ao desenvolvimento de O. ilicis e o aumento da temperatura, dentro da faixa ótima, poderá aumentar os problemas com essa espécie, pois poderá ocorrer maior número de geraçôes no período.

Portanto, são importantes os estudos que avaliem o impacto da alteração da temperatura no comportamento desta espécie, fornecendo dados que esclareçam melhor as condiçōes que favorecem seu desenvolvimento.

\section{REFERÊNCIAS}

APONTE, O.; McMURTRY, J.A. Biology, life table and mating behavior of Oligonychus perseae (Acari: Tetranychidae). International Journal of Acarology, v.23, p.199-207, 1997.

BONATO, O. The effect of temperature on life history parameters of Tetranychus evansi (Acari: Tetranychidae). Experimental and Applied Acarology, v.23, p.11-19, 1999.

BOUDREAUX, H.B. Biological aspects of some phytophagous mites. Annual Review of Entomology, v.8, p.137-154, 1963.

CENTRO CAPIXABA DE METEOROLOGIA E RECURSOS HÍDRICOS (CECAM). Série Histórica. Disponível em: http:// cecam.incaper.es.gov.br. Acesso em 30 de janeiro de 2010.

CONAB. Cafés do Brasil: safra 2008/2009. Brasília: MAPA/ CONAB, 2010. 130p.

FASSIO, L.H.; SILVA, A.E.S. Importância econômica e social do café conilon. In. FERRÃO, R.G.; FONSECA, A.F.A.; BRAGANÇA, S.M.; FERRÃO, M.A.G.; MUNER, L.H. DE. (Eds.). Café Conilon. Vitória: Incaper, 2007. p.37-52.

FAZUOLI, L.C.; THOMAZIELLO, R.A.; CAMARGO, M.B.P. Aquecimento global, mudanças climáticas e a cafeicultura paulista. O Agronômico, v.59, p.19-20, 2007.
FERREIRA, R.C.F.; OLIVERA, J.V.; HAJI, F.N.P.; GONDIM JR., M.G.C. Biologia, exigências térmicas e tabela de vida de fertilidade do ácaro-branco Polyphagotarsonemus latus (Banks) (Acari: Tarsonemidae) em videira (Vitis vinifera L.) cv. Itália. Neotropical Entomology, v.35, p.126-132, 2006.

FRANCO, R.A.; REIS, P.R.; ZACARIAS, M.S.; ALTOÉ, B.F.; PEDRO NETO, M. Dinâmica populacional de Oligonychus ilicis (McGregor, 1917) (Acari: Tetranychidae) em cafeeiro e de fitoseídeos associados a ele. Coffee Science, v.3, p.38-46, 2008.

HADDAD, M.L.; PARRA, J.R.P.; MORAES, R.C.B. Métodos para estimar os limites térmicos inferior e superior de desenvolvimento de insetos. Piracicaba: FEALQ/USP, 1999. 29p.

MATIELLO, J.B. O café: do cultivo ao consumo. São Paulo: Globo, 1991. 320p.

REIS, P.R. Ácaro-vermelho. Cultivar, v.7, p.14-17, 2005.

REIS, P.R.; SOUZA, J.C. Pragas do cafeeiro. In: RENA, A.B.; MALAVOLTA, E.; ROCHA, M.; YAMADA. T. (Ed.). Cultura do cafeeiro: Fatores que afetam a produtividade. Piracicaba: Associação Brasileira para Pesquisa da Potassa e do Fosfato, 1986. p.347-356.

REIS, P.R.; ZACARIAS, M.S. Ácaros em cafeeiro. Belo Horizonte: EPAMIG, 2007. 76p. (Boletim Técnico, 81)

REIS, P.R.; ALVES, E.B.; SOUSA, E.O. Biologia do ácarovermelho do cafeeiro Oligonychus ilicis (McGregor, 1917). Ciência e Agrotecnologia, v.21, p.260-266, 1997.

REIS, P.R.; PEDRO NETO, M.; FRANCO, R.A.; TEODORO, A.V. Controle de Brevipalpusphoenicis (Geijskes, 1939) e Oligonychus ilicis (McGregor, 1917) (Acari: Tenuipalpidae, Tetranychidae) em cafeeiro e o impacto sobre ácaros benéficos.I - abamectin e emamectin. Ciências e Agrotecnologia, v.28, p.269-281, 2004.

SILVA, C.A.D. Biologia e exigências térmicas do ácaro-vermelho (Tetranychus ludeni Zacher) em folhas de algodoeiro. Pesquisa Agropecuária Brasileira, v.37, p. 573-580, 2002.

VASCONCELOS, G.J.N.; DA SILVA, F.R.; GONDIM JR., M.G.C. BARROS, B.; OLIVEIRA, J.V. Efeito de diferentes temperaturas no desenvolvimento e reproduçáo de Tetranychus abacae Baker \& Printchard (Acari: Tetranychidae) em bananeira Musa sp. cv. Prata. Neotropical Entomology, v.33, p.149-154. 2004. 